\title{
Contraprueba terapéutica en el diagnóstico de la enfermedad celiaca. Metódica e indicaciones
}

DRES.: FRANCISCO LARRAIN", CARMEN FICUEROA*, OSVALDO DANUS *

La enfermedad celíaca es una entidad frecuente en la patología pediátrica de nuestro medio. Si bien es cierto el diagnóstico no ofrece dificultades cuando el cuadro estí plenamente desarrolliado, en el lactante o pre-escolar menor existen situaciones de difercnte índole que dificultan el diagnóstico.

Desde Iuego, en el lactante son frecuentes los procesos entéricos agudos que al repetirse pueden sinular un sindrome diarreico crónico; por otra parte es habitual la existencia de otras entidades condicionantes de malabsorción (como parasitosis y desnutrición), y el estudio histológico de mucosa intestinal, que es un elemento importante en el diagnóstico, no puede realizarse siempre en cl momento oportuno, debido a la demanda asistencial de estos problemas. Además, es corriente que la gravedad del enfermo no permita realizar el estadio pertinente y obligue al médico a iniciar el tratratamiento de enfermedad celíaca basado sólo en los antecedentes clínicos del niño.

Existe posteriormente la obligación de precisar el diagnóstico, ya que esta enfermedad somete al paciente a un régimen riguroso y prolongado, que se justifica sólo si existe un diagnóstico preciso. En este sentido la prueba y contraprueba terapéuticas adquieren su máximo valor.

No existen criterios ni pautas definidas que permitan catalogar el resultado de estas pruebas.

En la presente comunicación se expone un caso clínico que representa una metódica de prueba y contraprueba terapéuticas que sería de valor práctico y, que además, en cuestra experiencia ha tenido buen rendimiento.

Caso clínico. A. P. M. 1 año 3 meses. Sexo masculino. Lactante que ingresa al año tres me-

\footnotetext{
- Departamento de Pediatría. Hospital Roberto del Río.
}

ses de edad con el antecedente de haber presentado dos meses antes estomatitis de veinte días de evolución, continuando con inapetencia, y posteriormente diarrea y vómitos que habían cedido parcialmente al tratamiento ambulatorio.

El progreso ponderal había sido satisfactorio hasta los seis meses de edad, (6.750 grs.) observándose luego una frenación, de tal modo que a los doce tileses pesaba 8.600 grs.

A su ingreso se apreció en mal estado general y nutritivo (peso: 7.120 grs., talla: $74,6 \mathrm{cms}$.), edcma de extremidades inferiores y carla, signos carenciales de piel y distensión abdominal por meteorismo. El diagnóstico de ingreso fue desnutrición pluricarencial.

Una vez fundidos los edemias, el peso quedó en 6.340 grs. La evolución fue tótpida en los primeros 15 días, apreciándose deposiciones alteradas y en ocasiones veluminosas. Se descartó infección urinaria, parasitosis intestinal, y los coprocultivos fueron negativos. El hematocrito fue de $37 \%$, y la proteinemin de 5,2 grs. \%.

En base a fodos estos antecedentes clínicos, se sospechó una enfermedad celíaca, dejándose con régimen sin gluten. Se dio de alta a los 30 días sin diarrea, con 7.510 grs. de peso, indicándosele su control en Gastroenterología. En consideración a que el paciente había recibido un régimen libre de gluten, y experimentado una respuesta clínica favorable, se decidió continuar con dicho régimen, para realizar a continuación una contraprueba terapéutica, como método para precisar el diagnóstico.

Cinco meses después el peso fue de 10.900 grs. En este momento, el test de caroteno reveló un valor de 68 gamas \% (valor normal: sobre 50 gamas \%) y se decidió introducr el gluten en la 


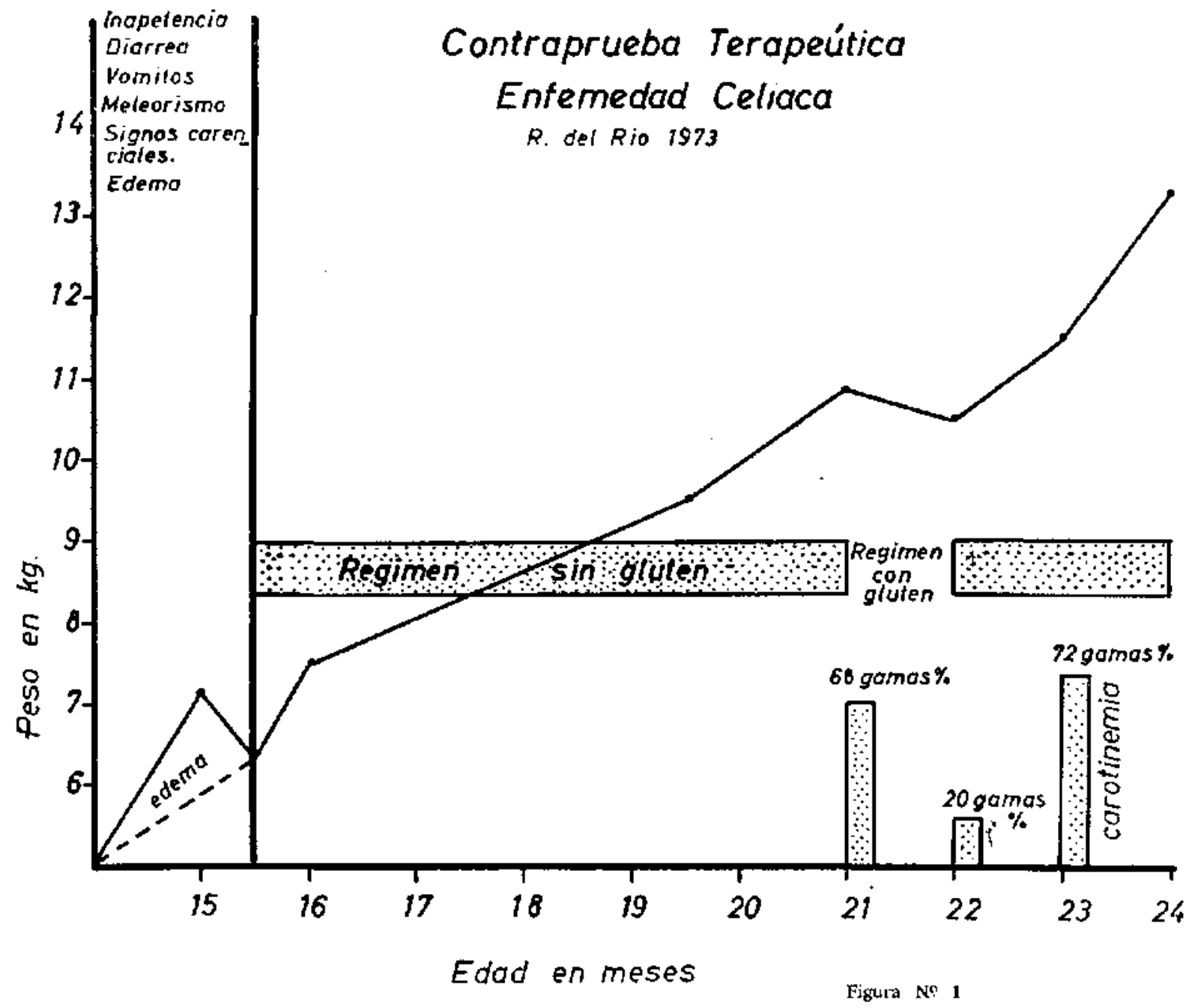

dicta, continuándose con controles mensuales, tanto clínicos como de Laboratorio. Un mes después apareció meteorismo abdominal, el peso descendió en 300 grs. $y$ el caroteno fue de 20 gamas $\%$. Se consideró la contraprueba como positiva y se decidió suspender definitivamente el gluten. Al mes siguiente, continuaba sin diarrea, el peso ascendió a 11.400 grs. $y$ el caroteno fue de 72 gamas $\%$. A los 2 años continuaba asintomático, pesaba 13.250 grs. y medía $88 \mathrm{~cm}$. (Fig. Nọ 1).

Comentario. De acuerdo con el criterio sustentado por la Sociedad Europea de Gastroenterología Pediátrica, el diagnóstico de la enfermedad celíaca se encuentra fundamentado cuando un sindrome de malabsorción presenta lesiones de atrofia vellocitaria subtotal en la mucosa intestinal, $y$ existe dependencia al gluten de la dieta (1).

Una vez iniciado el régimen libre de gluten los test de absorción y la histología intestinal presentan un valor diagnóstico relativo, ya que es posible

obtener mejoría importante de ellos en periodos tan cortos como de una semana (2), (3), (4).

En estas circunstancias, sólo la prueba y contraprueba terapéutica son de utilidad, siendo habitual que el resultado sea medido por la respuesta clínica del paciente a los cambios de la dieta. Sin embargo, la experiencia de otros autores (5), así como la muestra (6), señalan que las recaídas clínicas en la enfermedad celíaca son muy variables, tanto en el período de latencia que media entre la introducción del gluten y la reaparición de los síntomas, así como en las manifestaciones clínicas iniciales, las que con frecuencia plantean dudas en cuanto a su rclación con la enfermedad en estudio. Por otra parte, no parece lo más adecuado csperar el Lcterioro del paciente para confirmar el diagnóstico.

En las experiencias ya señaladas, ha podido apreciarse que cuando se agrega gluten en la dieta de un celíaco, se producen cambios importantes $y$ precoces en los tests, que miden absorción in- 
testinal, así como alteraciones estructurales de la mucosa, que demuestran el efecto deletéreo del gluten. Estas alteraciones anteceden, a veces por períodos de meses, a los primeros síntomas de la recaida. De ahí entonces que toda contraprueba deba evaluarse periódicamente por algunos de los tests mencionados.

Debido a los óptimos resultados obtenidos en nuestra experiencia con el test de caroteno plasmático (7) y la facilidad de empleo, se decidió utilizarlo como elemento de control en esta prueba.

En el caso presentado, las variaciones experimentadas por la carotinemia deben atribuirse exclusivamente al efecto del gluten sobre la mucosa intestinal, ya que durante ese período no existieron otros factores que pudieron interferir en el resultado, como sería la diarrea intercurrente, vómitos, hipoalimentación, o parasitosis.

\section{RESUMEN}

Se presenta un lactante de 1 año 3 meses de edad portador de enfermedad celíaca cuyo diagnóstico se basó en las variaciones de la carotinemia ante el retiro $y$ reintroducción del gluten en la dieta.

Se considera que la utilización de la prueba y contraprueba terapéutica como criterio diagnóstico de la enfermedad celiaca constituye una situación cada vez más corriente en la práctica pediátrica por la frecuencia con que se presenta esta entidad en nuestro medio y la imposibilidad de realizar un estudio inticial adecuado en gran parte de estos pacientes.

Hasta el momento no existen criterios definidos y prácticos que permitan estandarizar esta prueba. Por este motivo se sugiere el uso del test de caroteno plasmático, y se muestra su empleo, como parámetro útil en la evaluación del procedir miento ya que en nuestra experiencia ha mostrado una buena correlación tanto con la clinica como con la histología duodeno-yeyunal.

\section{SUMMARY}

The paper consider the utilization of the free gluten diet continued by a load gluten diet like a therapeutic proof of practic help for the celiac disease diagnosis. It suggest the employ of the plasmatic caroteno's test like a parameter with utility in the evaluation of the proceeding, that reveals a good correlation between the clinic and the duodeno-yeyunal histology.

\section{REFERENCLAS}

1.-Dahlquist, A.: Lindberg, T.; Meekwise, S.; Akerman, $M$. Intestinal Dipeptidases and Disaccharidases in Children with Malabsorption. Acta Paediat. Scand. 59: $621,1970$.

2.-Bayless, Th. M.: Yardley, J. H., Norton, J. H., Hendrix, Th. R. Adult Celiac Disease: Rapid sequential changes in Jeyunal Mucosa with alterations of dietary gluten. The J. of Clin. Invest. 41: 1344, 1962.

3.-Yardley, G. H., Bayless, Th. M.; Nonton, J. A.; Hendrix, Th. R. Celiac Disease. A study of the yeyunal epithelium before and after a gluten-free diet. The New England J. of Med. 267: 1173, 1962.

4.-Samloff, I. M,; Dovis, J. S.; Schenk, E. A. A clioical and histochemical study of celiac disease before and during a gluten-free diet. Gastroenterology 48: 155, 1965.

5.- Young, W. F.; Pringle, E. M. 110 Children with Coeliac Disease. 1950-1969. Arch. of Disease in Chilhood. 46: 421, 1971.

6.-Danús, O.; Chuaqui, B.; Solimano, G.; Urbina, A. M. Enteropatía sensible al gluten. Pediatría 13: 45, 1970.

7.-Danús, O.; Hormazábal, J. Test de caroteno plasinático en el diagnóstico de la enfermedad celíaca. Correlación con la histologia duodeno-yeyunal. Pediatría (en prensa). 